

\title{
Recent results and perspectives in heavy-ion physics
}

\author{
Raimond Snellings* \\ Nikhef \\ Kruislaan 409, \\ 1098 SJ Amsterdam, \\ The Netherlands \\ E-mail: Raimond.Snellings@nikhef.nl
}

Lattice QCD predicts a phase transition between hadronic matter and a system of deconfined quarks and gluons (the Quark Gluon Plasma) at high energy densities. Our current understanding of this new state of matter will be discussed with two key results from the Relativistic Heavy Ion Collider (RHIC).

8th Conference Quark Confinement and the Hadron Spectrum September 1-6 2008

Mainz, Germany

* Speaker. 


\section{Introduction}

Quantum Chromo Dynamics (QCD) provides, as part of the standard model, a very successful description of strong interaction processes involving large momentum transfer. The known QCD Lagrangian provides in principle the complete picture but the QCD field equations are notoriously hard to solve. In fact, the two most important and interesting properties, confinement and chiral symmetry breaking, are still poorly understood from first principles. Better understanding of these concepts can be obtained if we are able to study quarks and gluons in a deconfined state, the socalled Quark Gluon Plasma (QGP).

Relativistic heavy-ion collisions are considered a unique tool to produce the QGP to study deconfinement and the Equation of State (EOS) of hot QCD matter under controlled conditions. Therefore ultra relativistic heavy-ion experiments have been performed at the Brookhaven Alternating Gradient Synchrotron (AGS), the CERN Super Proton Synchrotron (SPS) and the Brookhaven Relativistic Heavy Ion Collider (RHIC) with maximum center of mass energies of $\sqrt{s_{\mathrm{NN}}}=4.75$, 17.2 and $200 \mathrm{GeV}$ respectively. The future Large Hadron Collider (LHC) will make $\mathrm{Pb}-\mathrm{Pb}$ collisions available at an unprecedented energy of $\sqrt{s_{\mathrm{NN}}}=5.5 \mathrm{TeV}$.

\section{Relativistic Heavy Ion Collisions}

A deconfined state of quarks and gluons might be created in the laboratory in heavy-ion collisions at the highest energies. Theoretical guidance for this comes from Lattice QCD calculations. Lattice QCD predicts that at an energy density $\varepsilon \approx 1 \mathrm{GeV} / \mathrm{fm}^{3}$, corresponding to a temperature of about $170 \mathrm{MeV}$, the system undergoes a phase transition from nuclear matter to a deconfined system of quarks and gluons. Figure 1a shows the calculated energy density as a function of tem-
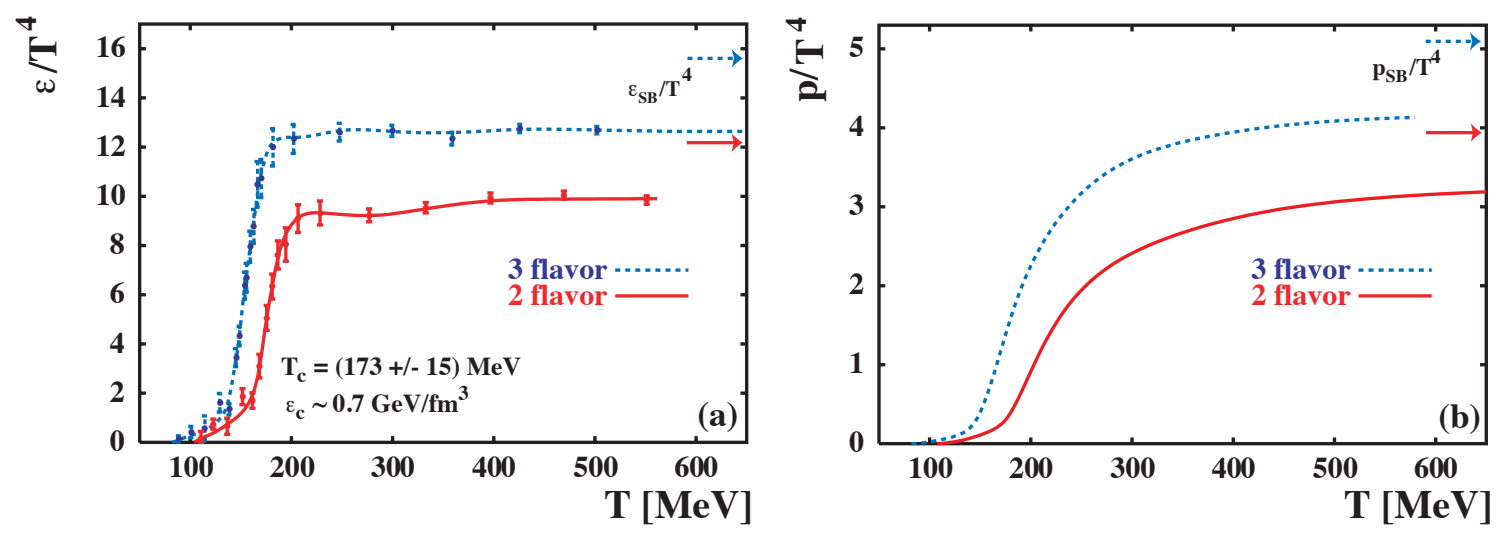

Figure 1: (Color on-line) a) Energy density $\varepsilon$ as a function of temperature from lattice calculations [1]. For an ideal gas the energy density is proportional to the number of thermal degrees of freedom. This causes the sharp increase at $T_{c}$ where the system goes from a pion gas, $g=3$, to a quark gluon plasma where $g=37$ in case of two quark flavors. The arrows in the figure indicate the ideal Stefan-Boltzmann values. b) The pressure from lattice calculations versus the temperature. The pressure also reflects the number of degrees of freedom but changes slowly at the phase boundary.

perature [1]. It is seen that the energy density changes rapidly at the critical temperature $T_{c} \sim 170$ 
$\mathrm{MeV}$, which is due to the rapid increase in the effective degrees of freedom. These lattice calculations indicate that at $T_{c}$ not only deconfinement sets in but that also chiral symmetry is restored. The pressure, shown in Fig. 1b, changes slowly at $T_{c}$ compared to the rapid increase of the energy density. Therefore the pressure gradient in the system, $\mathrm{d} P / \mathrm{d} \varepsilon$, is significantly reduced during the phase transition.

Like the early universe, the hot and dense system created in a heavy-ion collision will expand and cool down. In this time evolution the system probes a range of energy densities and temperatures, and possibly different phases. The evolution of the created system can be divided in a few characteristic periods. During the formation of the system $\left(\leq 3 \times 10^{-24} \mathrm{sec}\right)$ collisions with large momentum transfer occur. During this period the largest energy density is created. The system will thermalize and form the QGP provided that the quarks and gluons undergo multiple interactions. Due to the thermal pressure, the system undergoes a collective expansion and eventually becomes so dilute that it hadronizes. In the hadronic phase it further cools down via inelastic and elastic interactions until it becomes non-interacting (the freeze-out stage).

\section{High- $p_{t}$ observables}

The products of initial state hard scatterings, e.g. mesons containing charm or bottom, high- $p_{t}$ photons and high- $p_{t}$ hadrons provide a detailed probe of the created system. While initial state production of these probes is relatively unaffected by the presence of the created system it was predicted that partons lose energy while traversing the system, mainly by induced gluon radiation. The amount of energy loss in this picture is directly related to the parton density. Thus by studying these high- $p_{t}$ probes the density of the created system can be determined. In heavy-ion collisions at RHIC, jets with transverse energies above $40 \mathrm{GeV}$ are produced in abundance, however the abundant soft particle production in heavy-ion collisions tends to obscure the characteristic jet structures. At sufficient high- $p_{t}$ the contribution from the tails of the soft particle production becomes negligible and jets can be identified by their leading particles. When the parton loses energy, the hadrons in which it fragments will, on average, have lower transverse momenta. Therefore the parton energy loss leads to a suppression of the yield of high- $p_{t}$ hadrons compared to nucleon-nucleon collisions where there is no dense medium.
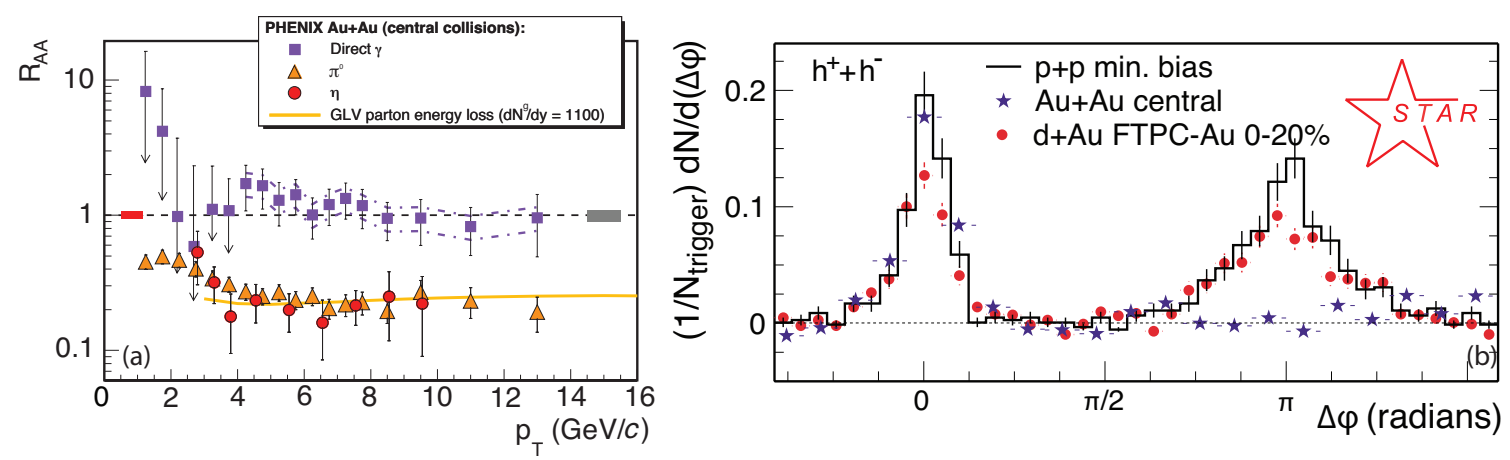

Figure 2: (Color on-line) a) The nuclear modification factor $R_{\mathrm{AA}}$ as function of transverse momentum for $\pi_{0}$ 's, $\eta$ 's and direct photons [2]. b) measured back to back leading hadron azimuthal correlations [3]. 
One of the observables suggested for measuring energy loss is the so called nuclear modification factor defined by

$$
R_{\mathrm{AA}}\left(p_{t}\right)=\frac{\mathrm{d}^{2} \sigma_{\mathrm{AA}} / \mathrm{d} y \mathrm{~d} p_{t}}{\left\langle N_{\text {binary }}\right\rangle \mathrm{d}^{2} \sigma_{\mathrm{pp}} / \mathrm{d} y \mathrm{~d} p_{t}},
$$

where $\mathrm{d}^{2} \sigma_{\mathrm{pp}} / \mathrm{d} y \mathrm{~d} p_{t}$ is the inclusive cross section measured in $\mathrm{p}+\mathrm{p}$ collisions and $\left\langle N_{\text {binary }}\right\rangle$ accounts for the geometrical scaling from $\mathrm{p}+\mathrm{p}$ to nuclear collisions. In the case that a $\mathrm{Au}+\mathrm{Au}$ collision is an incoherent superposition of $\mathrm{p}+\mathrm{p}$ collisions this ratio $R_{\mathrm{AA}}$ would be unity. Energy loss and shadowing would reduce this ratio below unity while anti-shadowing and the Cronin effect would lead to a value above unity. Figure $2 \mathrm{a}$ shows this ratio for $\pi_{0}$ 's and $\eta$ 's in central $\mathrm{Au}+\mathrm{Au}$ collisions at mid-rapidity [2]. The ratio is well below one and at high- $p_{t}$ the suppression is about a factor of 5 . In the same figure the $R_{\mathrm{AA}}$ for direct photons is shown. Direct photons do not couple via the strong force with the produced system and therefore, as expected, their $R_{\mathrm{AA}}$ at high- $p_{t}$ is compatible with unity.

The parton energy loss shows up even more dramatically in the azimuthal correlations as shown in Fig. 2b. Azimuthal correlations between two high- $p_{t}$ hadrons in a nucleon-nucleon collision exhibit strong peaks at 0 and $\pi$ because the two partons from a hard collision traverse the system back to back in azimuth. In the dense medium created in heavy-ion collisions the energy loss reduces the yield of high- $p_{t}$ particles; therefore the high- $p_{t}$ particles which do escape the system are on average produced close to the surface. The recoiling associated parton is maximally affected by the energy loss causing a suppression of the away-side hadron jet. This jet-quenching effect is clearly observed in the gold-gold measurements shown in Figure 2b [3].

The magnitude of the observed suppression at the top RHIC energy indicates, in the jet quenching picture, densities which are at least a factor 30 higher than in nuclear matter. To become more quantitative the energy loss mechanism and the response of the medium has to be understood in more detail.
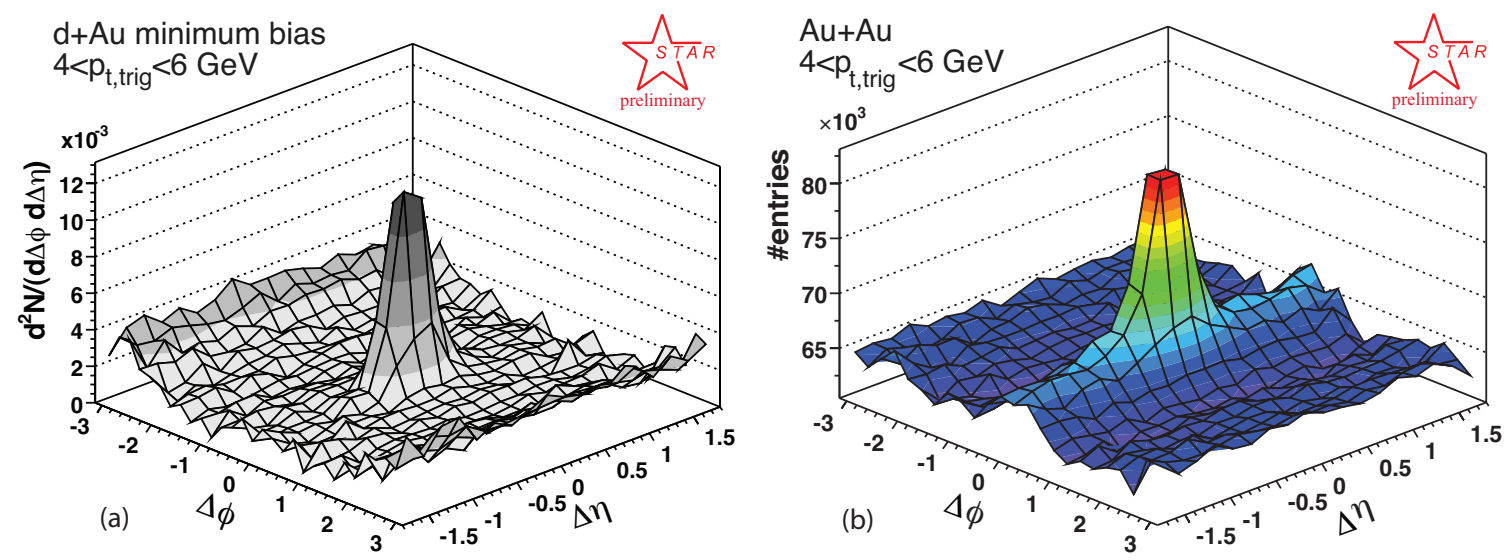

Figure 3: (Color on-line) Preliminary di-hadron correlations in $\Delta \eta$ and $\Delta \phi$ for a) d+Au and b) Au+Au. The trigger particle is between $4<p_{t}<6 \mathrm{GeV} / c$ and the associated particles are between $2 \mathrm{GeV} / c<p_{t}<$ $p_{t}^{\text {trig }}[4]$.

One recent striking result, which might provide additional information on the response of the medium, is the di-hadron correlation at intermediate $p_{t}$. The di-hadron correlation, in azimuth and 
in pseudo-rapidity $\eta$, shows that the near-side correlation (small $\Delta \phi$ which was already shown in Fig. 2b) extends over large $\Delta \eta$. Figure 3 shows this correlation for $\mathrm{d}+\mathrm{Au}$ and $\mathrm{Au}+\mathrm{Au}$ collisions [4]. It is clear that this longe range $\Delta \eta$ correlation is unique for heavy-ion collisions. The long range correlation is approximately independent of $\Delta \eta$ and is therefore referred to as the ridge.
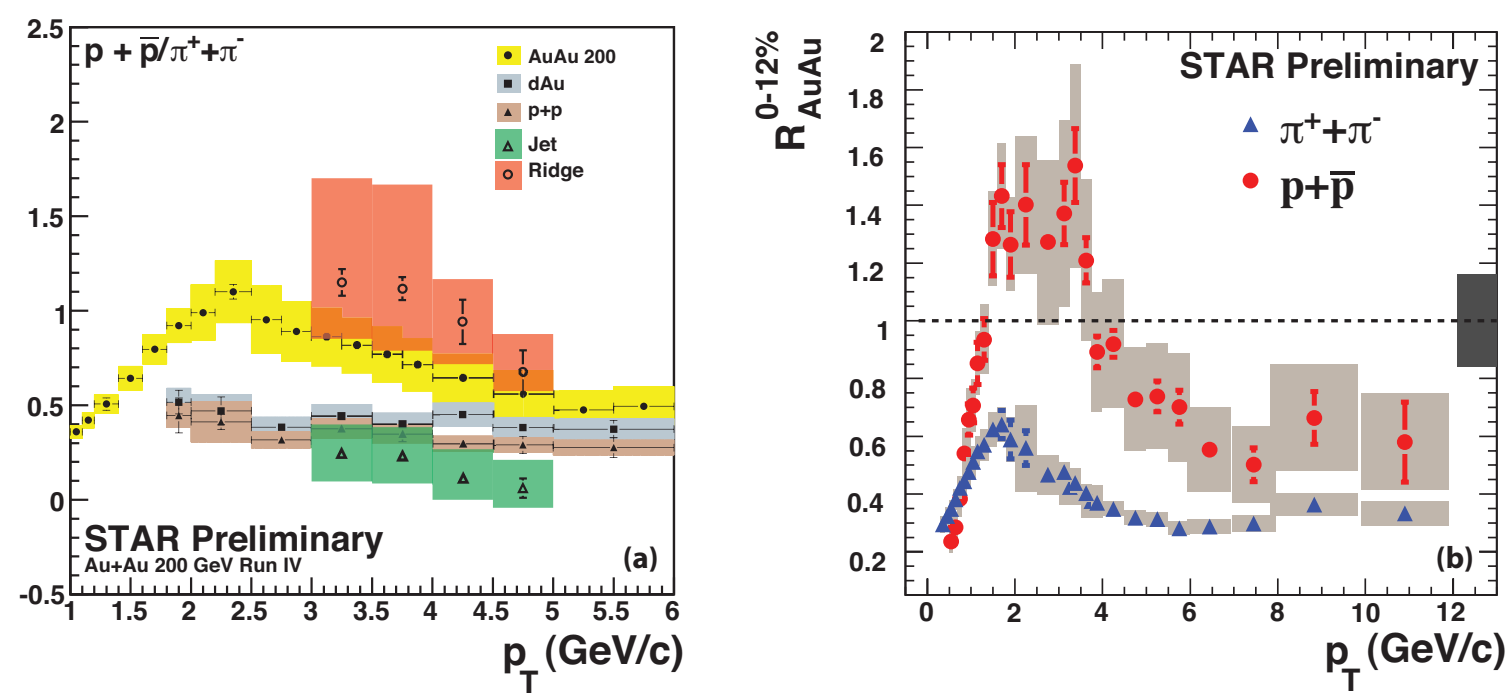

Figure 4: (Color on-line) a) Preliminary proton to pion ratio in the ridge and the jet-like peak in central $\mathrm{Au}+\mathrm{Au}$ collisions. For comparison the inclusive ratio in $\mathrm{p}+\mathrm{p}, \mathrm{d}+\mathrm{Au}$ and $\mathrm{Au}+\mathrm{Au}$ is shown. b) Preliminary nuclear modification factor $R_{\mathrm{AA}}$ for pions and protons in central $\mathrm{Au}+\mathrm{Au}$ collisions [5].

It is known that the particle composition at these intermediate $p_{t}$ 's is different between nucleonnucleon and heavy-ion collisions. Figure 4a shows this difference of more than a factor two in the proton to pion ratio for $\mathrm{p}+\mathrm{p}, \mathrm{d}+\mathrm{Au}$ and $\mathrm{Au}+\mathrm{Au}$ collisions. This difference can be used to test if the ridge is part of the jet or if it is the response of the medium. The figure clearly shows that the $\mathrm{p} / \pi$ ratio in the ridge is similar to the inclusive $\mathrm{p} / \pi$ ratio in $\mathrm{Au}+\mathrm{Au}$ and the $\mathrm{p} / \pi$ ratio in the jet is similar to the inclusive ratio in $\mathrm{p}+\mathrm{p}$. These results clearly indicate that the ridge is formed from the medium and not from the jet fragments.

In $\mathrm{p}+\mathrm{p}$ collisions, based on NLO pQCD calculations, the protons originate predominantly from gluon jets while charged pions have substantial contribution from quark jets. Gluons, due to the non-Abelian nature of QCD, are expected to lose more energy than quarks in the medium. Therefore, at high- $p_{t}$ the nuclear modification factor $R_{\mathrm{AA}}$ for pions and protons is expected to be different. To test the energy loss mechanism the measured nuclear modification factor $R_{\mathrm{AA}}$ for pions and protons is compared in Fig. 4b [5]. Surprisingly, the proton $R_{\mathrm{AA}}$, even at $p_{t}>6 \mathrm{GeV} / c$, is larger than the charged pion $R_{\mathrm{AA}}$ which would not be expected if the gluons indeed would lose more energy.

\section{Anisotropic Flow}

Flow is an ever-present phenomenon in nucleus-nucleus collisions, from low-energy fixedtarget reactions up to $\sqrt{s_{\mathrm{NN}}}=200 \mathrm{GeV}$ collisions at the Relativistic Heavy Ion Collider (RHIC), 
and is expected to be observed at the Large Hadron Collider (LHC). Flow signals the presence of multiple interactions between the constituents and is an unavoidable consequence of thermalization.

The usual theoretical tools to describe flow are hydrodynamic or microscopic transport (cascade) calculations. Flow depends in the transport models on the opacity, be it partonic or hadronic. Hydrodynamics becomes valid when the mean free path of particles is much smaller than the system size and allows for a description of the system in terms of macroscopic quantities. This gives a handle on the equation of state of the flowing matter and, in particular, on the value of the sound velocity [6]. In both types of models it may be possible to deduce from a flow measurement whether the flow originates from partonic or hadronic matter or from the hadronization process $[7,8,9]$.

A convenient way of characterizing the various patterns of anisotropic flow is to use a Fourier expansion of the triple differential invariant distributions [10]:

$$
E \frac{\mathrm{d}^{3} N}{\mathrm{~d}^{3} \mathbf{p}}=\frac{1}{2 \pi} \frac{\mathrm{d}^{2} N}{p_{\mathrm{t}} \mathrm{d} p_{\mathrm{t}} \mathrm{d} y}\left\{1+2 \sum_{n=1}^{+\infty} v_{n} \cos \left[n\left(\varphi-\Psi_{R}\right)\right]\right\},
$$

where $\varphi$ and $\Psi_{R}$ are the particle and reaction-plane azimuths in the laboratory frame, respectively. The sine terms in such an expansion vanish due to reflection symmetry with respect to the reaction plane. The Fourier coefficients are given by

$$
v_{n}\left(p_{\mathrm{t}}, y\right)=\left\langle\cos \left[n\left(\varphi-\Psi_{R}\right)\right]\right\rangle,
$$

where the angular brackets denote an average over the particles, summed over all events, in the $\left(p_{\mathrm{t}}, y\right)$ bin under study. In this parameterization, the first two coefficients, $v_{1}$ and $v_{2}$, are known as directed and elliptic flow, respectively.


Figure 5: (Color on-line) (a) Elliptic flow (solid points) as a function of centrality defined as $n_{c h} / n_{\max }$. The open rectangles show a range of values expected for $v_{2}$ in the hydrodynamic limit, scaled from $\varepsilon$, the initial space eccentricity of the overlap region. From [11] (b) Elliptic flow of pions and protons as function of transverse momentum [12]. The lines are hydrodynamical model calculations using two different Equations of State $(\mathrm{EoS})$, the dashed lines represent calculations done with a hadron gas EoS while the solid curves are calculation with an EoS which incorporates the QCD phase transition.

Elliptic flow has its origin in the amount of rescattering and the spatial eccentricity of the collision zone. The amount of rescattering is expected to increase with increasing centrality, while 
the spatial eccentricity decreases. This combination of trends dominates the centrality dependence of elliptic flow. The spatial eccentricity is defined by

$$
\varepsilon=\frac{\left\langle y^{2}-x^{2}\right\rangle}{\left\langle y^{2}+x^{2}\right\rangle}
$$

where $x$ and $y$ are the spatial coordinates in the plane perpendicular to the collision axis. The brackets \langle\rangle denote an average weighted with the initial density.

Figure 5a shows the first measurement of elliptic flow at RHIC [11]. Generally speaking, large values of elliptic flow are considered signs of hydrodynamic behavior as was first put forward by Ollitrault [6]. In hydrodynamics $v_{2}$ is essentially proportional to the spatial eccentricity (the strength depends on the velocity of sound of the matter). The open rectangles in Fig. 5a show, for a range of possible values of the velocity of sound, the expected $v_{2}$ values from ideal hydrodynamics. For $n_{c h} / n_{\max } \geq 0.5(\mathbf{b} \leq 7 \mathrm{fm})$ it is observed that the data is well described by ideal hydrodynamics.

The observed large amount of collective flow, in particular elliptic flow, is one of the main experimental discoveries at RHIC $[13,14,15,16]$ and the main evidence suggesting nearly perfect fluid properties of the created matter [17].

Figure $5 \mathrm{~b}$ shows $v_{2}$ for identified particles as function of transverse momentum. At low $p_{\mathrm{t}}$ the elliptic flow depends on the mass of the particle with $v_{2}$ at a fixed $p_{\mathrm{t}}$ decreasing with increasing mass. This dependence is expected in a scenario where all the particles have a common radial flow velocity as shown by the curves in Fig. $5 \mathrm{~b}$ from ideal hydrodynamics. The difference between the dashed and solid curves is the EoS, the dashed curves correspond to calculations done with a hadron resonance gas EoS while the solid curves are hydro calculations incorporating the QCD phase transition. The sensitivity to the EoS is better for the heavier particles because they are less affected by the contribution of the finite freeze-out temperature.
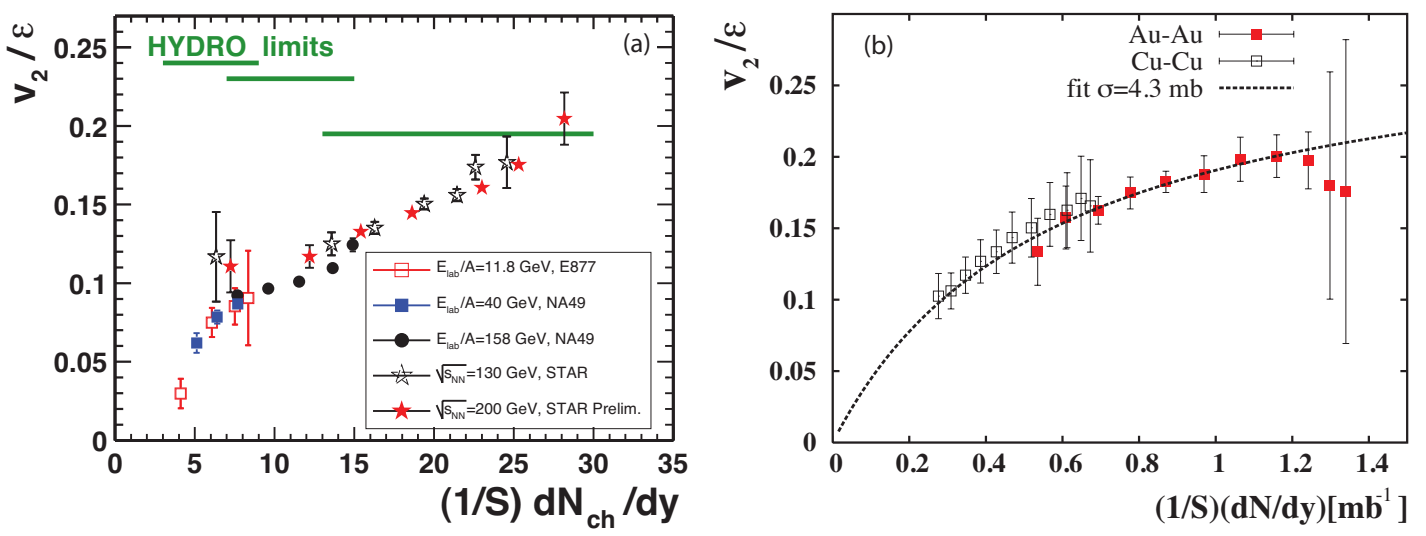

Figure 6: (Color on-line) (a) Compilation of $v_{2} / \varepsilon$ data [19] versus particle density at midrapidity. Green lines indicate ideal hydrodynamic predictions for AGS, SPS and RHIC collisions energies (b) Fit to $v_{2} / \varepsilon$ versus particle density in terms of Knudsen number for $\mathrm{Cu}+\mathrm{Cu}$ and $\mathrm{Au}+\mathrm{Au}$ [29]. The data are from PHOBOS.

As mentioned, the centrality dependence of elliptic flow is governed by the spatial eccentricity and particle density in the transverse plane [18]. This can be quantified by plotting $v_{2} / \varepsilon$ versus particle density in the transverse plane, $1 / S\left(d N_{c h} / d y\right)$, where the initial overlap area $S$ and eccentricity 
are taken from Glauber model calculations. This offers the possibility to compare results obtained at different collision energies, with different projectiles, and at different centralities. Non-smooth dependence would indicate new physics (e.g. deconfinement) and saturation could signal ideal hydrodynamical evolution. Figure 6a shows NA49 results [19] together with results obtained at the AGS and preliminary results from RHIC. The figure shows a continuous rise of $v_{2} / \varepsilon$ reaching the ideal hydrodynamic expectations (shown by green lines) only in the most central collisions at RHIC energies.

The ideal hydrodynamical expectations (green lines) for $v_{2} / \varepsilon$ are almost constant at fixed center of mass energy. The clear deviation of the data from this ideal hydro prediction is evidence that for more peripheral collisions and for energies below the highest RHIC energy the elliptic flow has significant non-ideal hydro contributions. Much of this discrepancy was explained by incorporating viscous contributions of the hadronic phase [20, 21, 22, 23]. However, more recently, Kovtun, Son and Starinets [24], showed that conformal field theories with gravity duals have a ratio of viscosity $\eta$ to entropy density $s$ of $1 / 4 \pi$ (in natural units). They conjectured that this value is a bound for any relativistic thermal field theory. Additionally, Teaney [25] pointed out that already very small viscosities would lead to a significant reduction in the predicted elliptic flow.

Currently there are two promising approaches to quantify how big the possible discrepancy with ideal hydrodynamics is and extract $\eta / s$. The first approach is to match the data using hydrodynamical models which incorporate viscous corrections [26, 27]. One of the drawbacks of this approach is that $\eta / s$ is not the only unknown, also the initial conditions and EOS need to be varied. The second approach is a fit of $v_{2} / \varepsilon$ versus particle density based on a parameterization in terms of the Knudsen number [28, 29]. The Knudsen number $K$ is the mean free path of the constituents divided by the system size. The fit yields $K$ and extrapolating the fit to $K=0$ yields the ideal hydrodynamical limit of $v_{2} / \varepsilon$. The latter defines the effective velocity of sound and thus the effective EOS. The authors conclude from the fit to the data, shown in Fig. 6b, that at RHIC we still might be up to $30 \%$ below the ideal "hydro limit" even for the most central collisions.

\section{Conclusions}

At RHIC energies all the observables indicate the creation of a very dense and strongly interacting system. This created system exhibits remarkable properties which pose many new questions. The presented recent progress on parton energy loss and anisotropic flow is bringing us closer to a quantitative description of this system.

\section{References}

[1] F. Karsch and E. Laermann. R.C. Hwa, editor, Quark gluon plasma, 1-59. 2003.

[2] S. S. Adler et al. [PHENIX Collaboration], Phys. Rev. C 75 (2007) 024909

[3] C. Adler et al. [STAR Collaboration], Phys. Rev. Lett. 90 (2003) 082302

[4] M. van Leeuwen [STAR collaboration], arXiv:0808.4096 [nucl-ex].

[5] B. Mohanty [STAR Collaboration], J. Phys. G 35 (2008) 104006

[6] J. Y. Ollitrault, Phys. Rev. D 46 (1992) 229. 
[7] P. Danielewicz, Nucl. Phys. A 661 (1999) 82.

[8] D. H. Rischke, Nucl. Phys. A 610 (1996) 88C.

[9] J. Y. Ollitrault, Nucl. Phys. A 638 (1998) 195.

[10] S. Voloshin and Y. Zhang, Z. Phys. C 70 (1996) 665.

[11] K. H. Ackermann et al. [STAR Collaboration], Phys. Rev. Lett. 86 (2001) 402

[12] C. Adler et al. [STAR Collaboration], Phys. Rev. Lett. 87 (2001) 182301

[13] I. Arsene et al. [BRAHMS Collaboration], Nucl. Phys. A 757 (2005) 1

[14] B. B. Back et al. [PHOBOS Collaboration], Nucl. Phys. A 757 (2005) 28

[15] J. Adams et al. [STAR Collaboration], Nucl. Phys. A 757 (2005) 102

[16] K. Adcox et al. [PHENIX Collaboration], Nucl. Phys. A 757 (2005) 184

[17] T.D. Lee et al., New Discoveries at RHIC: Case for the Strongly Interacting Quark-Gluon Plasma. Contributions from the RBRC Workshop held May 14-15, 2004. Nucl. Phys. A 750 (2005) 1-171

[18] H. Heiselberg and A. M. Levy, Phys. Rev. C 59, 2716 (1999)

[19] C. Alt et al. [NA49 Collaboration], Phys. Rev. C 68 (2003) 034903

[20] D. Teaney, J. Lauret and E. V. Shuryak, arXiv:nucl-th/0110037.

[21] D. Teaney, J. Lauret and E. V. Shuryak, Phys. Rev. Lett. 86 (2001) 4783.

[22] T. Hirano and M. Gyulassy, Nucl. Phys. A 769 (2006) 71

[23] T. Hirano, U. W. Heinz, D. Kharzeev, R. Lacey and Y. Nara, Phys. Lett. B 636 (2006) 299

[24] P. Kovtun, D. T. Son and A. O. Starinets, Phys. Rev. Lett. 94 (2005) 111601

[25] D. Teaney, Phys. Rev. C 68 (2003) 034913

[26] H. Song and U. W. Heinz, Phys. Rev. C 78, 024902 (2008) [arXiv:0805.1756 [nucl-th]].

[27] M. Luzum and P. Romatschke, Phys. Rev. C78, 034915 (2008)

[28] R. S. Bhalerao, J. P. Blaizot, N. Borghini and J. Y. Ollitrault, Phys. Lett. B 627 (2005) 49

[29] H. J. Drescher, A. Dumitru, C. Gombeaud and J. Y. Ollitrault, Phys. Rev. C 76 (2007) 024905 